\title{
Mode and dynamics of vanA-type vancomycin resistance dissemination in Dutch hospitals
}

\author{
Sergio Arredondo-Alonso ${ }^{1,2}$, Janetta Top ${ }^{1}$, Jukka Corander ${ }^{2,3,4}$, Rob J. L. Willems ${ }^{1}$ and Anita C. Schürch ${ }^{1 *}$
}

\begin{abstract}
Background: Enterococcus faecium is a commensal of the gastrointestinal tract of animals and humans but also a causative agent of hospital-acquired infections. Resistance against glycopeptides and to vancomycin has motivated the inclusion of E. faecium in the WHO global priority list. Vancomycin resistance can be conferred by the vanA gene cluster on the transposon Tn1546, which is frequently present in plasmids. The vanA gene cluster can be disseminated clonally but also horizontally either by plasmid dissemination or by $\mathrm{Tn} 1546$ transposition between different genomic locations.

Methods: We performed a retrospective study of the genomic epidemiology of 309 vancomycin-resistant $E$. faecium (VRE) isolates across 32 Dutch hospitals (2012-2015). Genomic information regarding clonality and Tn1546 characterization was extracted using hierBAPS sequence clusters (SC) and TETyper, respectively. Plasmids were predicted using gplas in combination with a network approach based on shared k-mer content. Next, we conducted a pairwise comparison between isolates sharing a potential epidemiological link to elucidate whether clonal, plasmid, or Tn1546 spread accounted for vanA-type resistance dissemination.
\end{abstract}

Results: On average, we estimated that $59 \%$ of VRE cases with a potential epidemiological link were unrelated which was defined as VRE pairs with a distinct Tn1546 variant. Clonal dissemination accounted for $32 \%$ cases in which the same SC and Tn1546 variants were identified. Horizontal plasmid dissemination accounted for $7 \%$ of VRE cases, in which we observed VRE pairs belonging to a distinct SC but carrying an identical plasmid and Tn1546 variant. In $2 \%$ of cases, we observed the same Tn1546 variant in distinct SC and plasmid types which could be explained by mixed and consecutive events of clonal and plasmid dissemination.

Conclusions: In related VRE cases, the dissemination of the vanA gene cluster in Dutch hospitals between 2012 and 2015 was dominated by clonal spread. However, we also identified outbreak settings with high frequencies of plasmid dissemination in which the spread of resistance was mainly driven by horizontal gene transfer (HGT). This study demonstrates the feasibility of distinguishing between modes of dissemination with short-read data and provides a novel assessment to estimate the relative contribution of nested genomic elements in the dissemination of vanA-type resistance.

Keywords: Enterococcus faecium, Genome sequencing, Vancomycin resistance, Network, Clonal dissemination, Horizontal dissemination, Horizontal gene transfer

\footnotetext{
* Correspondence: a.c.schurch@umcutrecht.nl

'Department of Medical Microbiology, University Medical Center Utrecht, Utrecht, The Netherlands

Full list of author information is available at the end of the article
}

(C) The Author(s). 2021 Open Access This article is licensed under a Creative Commons Attribution 4.0 International License, which permits use, sharing, adaptation, distribution and reproduction in any medium or format, as long as you give appropriate credit to the original author(s) and the source, provide a link to the Creative Commons licence, and indicate if changes were made. The images or other third party material in this article are included in the article's Creative Commons licence, unless indicated otherwise in a credit line to the material. If material is not included in the article's Creative Commons licence and your intended use is not permitted by statutory regulation or exceeds the permitted use, you will need to obtain permission directly from the copyright holder. To view a copy of this licence, visit http://creativecommons.org/licenses/by/4.0/ The Creative Commons Public Domain Dedication waiver (http://creativecommons.org/publicdomain/zero/1.0/) applies to the data made available in this article, unless otherwise stated in a credit line to the data. 


\section{Background}

Enterococcus faecium is commonly inhabiting the gut of animals and humans but has also emerged as a nosocomial pathogen causing a sizable fraction of healthcareassociated infections, specifically device-associated infections like central line-associated bloodstream and surgical site infections [1, 2]. The intrinsic and acquired multi-drug resistance against fluoroquinolones, aminoglycosides, and more importantly against glycopeptides motivated the inclusion of E. faecium in the WHO global priority list [3]. The number of strains resistant against vancomycin, a first-line glycopeptide antibiotic to treat infections with multi-drug-resistant Grampositive pathogens, dramatically increased first in the USA in the 1990s, followed by other parts of the world [4]. Resistance against vancomycin can be acquired through eight different gene clusters (vanA, vanB, vanD, vanE, vanG, vanL, vanM, and vanN) [5, 6] of which $v a n A$ and $v a n B$, associated to transposon sequences $\operatorname{Tn} 1546$ and $\operatorname{Tn} 1549$, respectively, are the predominant vancomycin resistance gene clusters [7].

Clonal spread of vancomycin-resistant Enterococcus faecium (VRE) has been extensively described using a plethora of molecular typing schemes. They range from fingerprint-based methods like pulsed-field gel electrophoresis [8] to PCR-based methods such as multiplelocus variable number tandem repeat analysis [9], multilocus sequence typing [10], and whole-genome sequencing [11]. However, due to the fact that vancomycin resistance genes are located on mobile genetic elements, vancomycin resistance also has the potential to be transferred horizontally. In fact, mobilization of the vanA gene cluster via insertion in different plasmid backbones has already been reported [12, 13]. To identify the dissemination of $v a n A$ plasmids within hospital settings, whole-genome sequencing (WGS) based on short-read technologies has been recently applied to collections of hundred hospitalized patient isolates in Denmark and Australia [14, 15]. These studies undertook a referencebased approach to map short-reads against complete plasmids from a selection of isolates. However, this approach can overestimate the presence of a reference plasmid by neglecting the mosaicism observed in these types of sequences as previously observed for Enterobacteriaceae isolates [16] and Enterococcus populations [17].

The dissemination of the vanA gene cluster can occur vertically, in which case the same plasmid type and Tn1546 variant are observed in two clonal isolates. However, the vanA gene cluster can also be horizontally transferred, by two different processes: (i) plasmid dissemination which is reflected by observing the same plasmid type and Tn1546 variant in strains that have a different clonal background and (ii) transposition of Tn1546 between different plasmid types [18-20]. This nested nature of these mobile genomic elements resembles the Russian-Doll model which has been previously used to describe the transfer of carbapanamese genes, bla $_{\mathrm{kpc}}$, in Enterobacteriaceae [16]. It is important to note that the $\operatorname{Tn} 1546$ is a non-conjugative transposon but its mobilization can occur when the element is embedded in another conjugative element. Furthermore, the presence of IS elements (e.g., IS1216) surrounding the transposon can mobilize the vanA gene cluster to other genomic locations [18-20].

The genomic approach conducted here allowed us to fully reconstruct and quantify the most likely mode of dissemination by characterizing the clonal background (hierBAPS SC), vanA plasmid type (de novo prediction and network assignment), and Tn1546 variants harboring the vanA resistance gene cluster. Clonal dissemination, defined by vertical inheritance of the same SC, vanA plasmid type, and $\operatorname{Tn} 1546$ variant, was the most frequent scenario of vancomycin resistance dissemination occurring in the Netherlands between 2012 and 2015. However, we also detected outbreak settings in which Tn1546 transposition between distinct plasmid types and/or plasmid dissemination were the dominant mechanisms driving the vanA gene cluster dissemination. To our knowledge, we provide one of the first studies to estimate the frequencies of clonal and HGT processes in the dissemination of the vanA gene cluster occurring in Dutch healthcare settings between 2012 and 2015.

\section{Methods}

\section{Dutch VRE collection, short-read WGS, and genome} assembly

The isolates from this collection represent a subset of isolates from a previous study we conducted and that consisted of 1644 E. faecium isolates [21]. Isolates with the vanA-type vancomycin resistance gene cluster $(n=$ 309) from 32 Dutch hospitals collected between 2012 and 2015 were further analyzed. DNA extraction and whole-genome sequencing using Illumina NextSeq were conducted as previously described [22]. Short-reads were trimmed using Trim Galore (version 0.6.4_dev) using the flag "--paired" and specifying a phred score of 20 with the flag "--quality" [23]. We used Unicycler (version 0.4.7) passing the short paired-end trimmed reads from Trim Galore [23], a wrapper script that combines cutadapt and fastqc [24, 25]. Unicycler was run specifying the normal mode (--mode) [26]. Unicycler was used to compute the assembly graph provided in the file "assembly.gfa" which selects for the k-mer size that optimizes the ratio between number of dead-ends and contig size in the graph given by SPAdes (version 3.14.0) [27]. For six isolates (E7931, E7942, E8155, E8387, E8437, E9000), Unicycler failed to generate a short-read assembly. In 
silico prediction using Abricate (https://github.com/ tseemann/abricate, version 0.8), with the ResFinder database (indexed on 16th of July 2018) [28] was conducted to search and select for isolates bearing the vanA resistance gene.

\section{Population structure of VREfm isolates}

Recombination events and estimation of sequence clusters, in the 309 VRE isolates, were performed using BratNextGen and hierBAPS and retrieved from ArredondoAlonso et al. [21]. PopPUNK (version 2.0.1) was run specifying the flag "--easy-run" with a minimum k-mer size of 13 (flag --min-k) and creating the files required to generate a microreact project (flag --microreact) [29].

\section{De novo plasmid prediction}

Gplas (version 0.6.1) was used to de novo predict the plasmids present in the assembly graph of each VRE isolate [30]. Gplas was run using mlplasmids [22] as a classifier to predict plasmid sequences (flag "-c"), specifying the species model "Enterococcus faecium" (flag -s), a modularity threshold of 0.1 to partition the resulting bins (flag -q), and 50 walks per plasmid seed (flag -x).

\section{Tn1546 characterization}

TETtyper (unique version) was used [31] to detect SNPs and deletions present in the $\operatorname{Tn} 1546$ sequences of each complete plasmid sequence or predicted vanA plasmid bin against a reference sequence (--ref) corresponding to the original transposon structure (NCBI Nucleotide Accession M97297) described by Arthur et al. [32]. We passed the trimmed reads to TETyper with otherwise default parameters.

\section{Network of complete plasmid sequences and plasmid type definition}

Mash (version 2.2.2) [33] specifying a k-mer size (-k) of 21 and sketch size (-s) of 1000 was used to perform kmer pairwise comparisons between complete plasmid sequences carrying the vanA gene cluster. For this purpose, we included the 26 complete vancomycin-resistant (vanA) plasmids from the same collection of 1644 E. faecium isolates [21] and 60 complete plasmid sequences carrying the Tn1546 (NCBI Nucleotide Accession M97297) from the PLSDB database [34]. Sequences were retrieved from the PLSDB database with the function Mash screen $(\max . p$ value $=0.1$, Min. Identity $=0.99$ ) We removed PLSDB sequences already present in our set of 26 complete vancomycin-resistant plasmids.

Based on the density distribution of Mash distances, we estimated an optimal cutoff of 0.025 to define the minimum distance to draw an edge between two nodes (complete plasmid sequences) in a network. The igraph $\mathrm{R}$ package (version 1.2.4) was considered to represent the network [35]. Independent components (subgraphs, size $>1$ node) in the network were considered as plasmid types $(\mathrm{A}-\mathrm{H})$. Singleton sequences in the network (NC_014726.1, AP022823.1, NC_013317.1, NZ CP012594.1, NC_005054.1, NZ_CP022486.1, NZ CP014531.1, NZ_CP040238.1, E8172_3, NZ CP036247.1, NZ_CP018130.1, NZ_CP019973.1) were not considered to define plasmid types but were included in all subsequent analyses.

To perform average nucleotide identity (ANI) measures between the complete plasmid sequences, we used the script "average_nucleotide_identity.py" provided in the pyani tool (version 0.2.10) [36] with default parameters. We further considered the reported ANIm alignment coverage and ANIm identity values to support plasmid type assignments. We used the ward.D2 method implemented in the function hclust of the stats $\mathrm{R}$ package (version 3.5.1) to cluster the ANIm alignment coverage. Next, we used the function heatmap. 2 from the gplots $\mathrm{R}$ package (version 3.0.1.1) to integrate the plasmid types previously defined using our proposed network approach with the clustering based on the ANIm alignment coverage.

For visualization purposes, the starting coordinate position of complete vanA plasmids was adjusted using the function fixstart from circlator (version 1.5.5) using a customized database of known plasmid replication initiator sequences [37]. Easyfig (version 2.2.2) [38] with a minimum $80 \%$ identity and minimum block length of $500 \mathrm{bp}$ were considered to visualize the blastn alignment [39] produced using the complete plasmid sequences belonging to the plasmid type $B$.

\section{Network of predicted plasmid bins and integration of plasmid types}

The predicted plasmid bins reported by gplas and bearing the $v a n A$ gene cluster were pairwise compared using Mash $(k=21, s=1000)$. The igraph $\mathrm{R}$ package (version 1.2.4) was used to represent a network in which nodes corresponded to vanA plasmid bins and edges to connections between bins with a Mash distance lower than 0.025 . The same threshold (0.025) to define an edge was considered since the density distribution of Mash distances followed the same pattern as previously observed with the complete plasmid sequences. Furthermore, we computed the total number of components and the average component size using different thresholds to estimate an optimal edge cutoff. The network consisted of 270 nodes and 16 independent components (subgraphs with $>1$ node). Component 3 (144 nodes) was partitioned into 3 different graph groups based on its modularity value (0.42) using the function "cluster_louvain" from the igraph $\mathrm{R}$ package (version 1.2.4). We focused 
on components/graph bins with $>10$ isolates that were termed as plasmid bin groups $(1-8)$.

Next, we integrated the plasmid types (A-I) and the singleton sequences (NC_014726.1, AP022823.1, NC_ 013317.1, NZ_CP012594.1, NC_005054.1, NZ_ CP022486.1, NZ_CP014531.1, NZ_CP040238.1, E8172_ 3, NZ_CP036247.1, NZ_CP018130.1, NZ_CP019973.1) into the network of predicted plasmid bins. Then, we computed Mash distances $(k=21, s=1000)$ between complete plasmid sequences and predicted plasmid bins. The igraph $\mathrm{R}$ package (version 1.2.4) was used to represent a network in which nodes either corresponded to $v a n A$ plasmid bins or complete plasmid sequences and edges to connections between sequences (bins or complete sequences) with a Mash distance lower than 0.025 . The network consisted of 348 nodes: 270 predicted plasmid bins and 78 complete plasmid sequences. Plasmid bin groups with edges connecting to complete plasmid sequences were assumed to carry the same plasmid type (A, B, C, D, E, I). Only the plasmid bin group J presented no edges connecting to complete plasmid sequences and it was considered as carrying a novel plasmid type.

\section{Contribution of nested genomic elements in the dissemination of vanA-type gene cluster}

Pairwise comparisons (e.g., 10 isolates, 45 unique pairs) were computed between VRE isolates sampled within 12 consecutive months and isolated: (i) country-wide, (ii) at the same Dutch region, and (iii) at the same hospital. To avoid grouping the isolates based exclusively on their isolation year, we computed all the windows of 12 months between two consecutive years (e.g., January 2012-January 2013, ..., December 2012-December 2013). Only windows with more than 10 isolates were considered to estimate the frequency of vanA dissemination. For each remaining window, we determined which genomic elements were shared between pairs of VRE isolates and defined the following scenarios: (i) clonal dissemination, characterized by identical SC, vanA plasmid type, and Tn1546 structure; (ii) HGT plasmid dissemination, characterized by identical vanA plasmid type and $\operatorname{Tn} 1546$ structure but distinct SC type; (iii) clonal spread mediated by $\operatorname{Tn} 1546$ mobilization between plasmid types co-existing in the same $\mathrm{SC}$, characterized by identical SC and Tn1546 structure but distinct plasmid type; (iv) Tn1546 mobilization between distinct SC and plasmid types, characterized by distinct SC and plasmid types but identical Tn1546 structure; and (v) no linkage (unrelated cases), distinct Tn1546 structure. For every two consecutive years (2012-2013, 2013-2014, 20142015), we averaged the frequency of the observed scenarios in each of the windows (> 10 isolates).

\section{Visualization of genomic elements}

GADM (version 3.6) [39] was used to download the map with regional borders of the Netherlands and was combined with the $\mathrm{R}$ packages ggplot2 (version 3.1.0) and scatterpie (version 0.1.5) (https://github.com/ GuangchuangYu/scatterpie) to plot spatial information and SC distribution of the isolates. The R package genoplotR (version 0.8.9) [40] was used to visualize the gene structure and to highlight the vanA gene cluster location and the presence of IS elements present in the plasmid types (A-F). The R package ggtree (version 1.14.6) [41] was used to integrate the neighbor-joining tree based on the core genome given by PopPUNK together with SC assignment and predicted vanA plasmid types. In addition, a Microreact project (version 15.0.0) [42] was created to integrate and visualize the genomic and metadata information.

An RMarkdown document is provided to integrate all the code and reproduce the results generated for all the methods section described above [43].

\section{Results}

The population structure of VRE from Dutch hospitals

This study was conducted with samples from an extensive collection of 1644 E. faecium isolates derived from clinical and non-clinical sources with associated short-read WGS data [21]. We focused on Dutch clinical isolates with complete metadata information regarding clinical settings and isolation date, from 2012 to $2015(n=593)$. From this selection, 309 $(52.1 \%)$ and $265(44.7 \%)$ isolates carried the vanA and/or $v a n B$ gene clusters, respectively. We focused on the vanA VRE samples since the resistance gene cluster is frequently present on plasmids [44, 45]. This permitted us to investigate a nested genomic system in which the glycopeptide resistance can be disseminated on a clonal, plasmid, and/or transposon level. These 309 VRE samples were isolated from patients in 32 Dutch hospitals.

The clonality of these 309 vanA VRE samples was determined using hierBAPS [46], after filtering for recombination events as previously described [21]. HierBAPS defined 18 different sequence clusters (SCs) of which SC13 $(n=102,33 \%), \operatorname{SC} 17(n=52$, $16.8 \%)$, and $\mathrm{SC} 18(n=42,13.6 \%)$ represented the most predominant clones in the dataset (Fig. 1a). The distribution of these SC across time and geographical position showed that $\mathrm{SC} 13$ was widespread in Dutch hospitals for the entire collection period (2012-2015) (Fig. 1b) compared to SC17 which was observed in distinct regions (North-Holland, Flevoland, Overijssel) from August-September 2012 (Fig. 1b). SC18 was detected around 2014 and 2015 in several Dutch regions (Fig. 1b). We computed a 


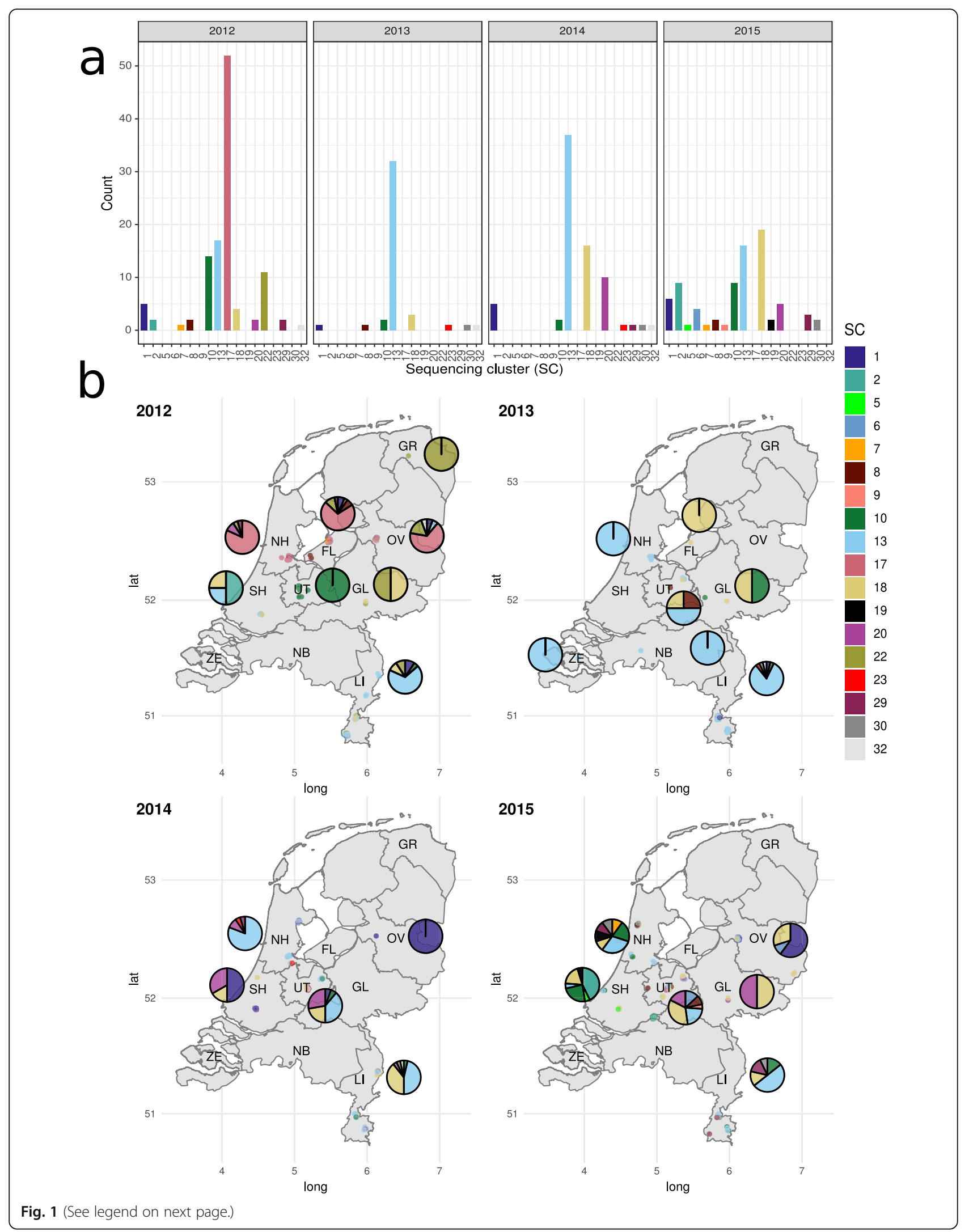


(See figure on previous page.)

Fig. 1 Temporal and spatial distribution of the 309 VRE isolates per isolation year (2012-2015). Isolates were colored according to their associated hierBAPS sequencing clusters (SC) $(n=18)$. a Count of the total number of isolates grouped in each hierBAPS sequencing cluster (SC). $\mathbf{b}$ For each isolate, the latitude and longitude of the hospital where the sample was processed are indicated. To mitigate overlapping, the points representing the isolates were jittered and their specific coordinates can be inspected at https://microreact.org/project/FCUD_d1zt. For each region, a pie chart showing the distribution of SCs is indicated to facilitate the inspection of the results. The names of the Dutch regions with isolates present are abbreviated in the following manner: FL Flevoland, GL Gelderland, GR Groningen, LI Limburg, NB North-Brabant, NH NorthHolland, OV Overijssel, SH South-Holland, UT Utrecht, ZE Zeeland

core-genome-based neighbor-joining tree of the samples using PopPUNK [29] for 303 isolates (98.1\%) with an associated short-read assembly [29], and the tree was combined with metadata information in the following Microreact project https://microreact.org/ project/FCUD_d1zt. Metadata information and SC assignment of all VRE isolates $(n=309)$ are also available in Additional File 1: Table S1.

Table 1 Metadata information of the isolates carrying the vanA complete plasmid sequences $(n=25)$ described in ArredondoAlonso et al. [21] and used to define the plasmid types: A, B, C, D, E, F

\begin{tabular}{|c|c|c|c|c|c|c|c|}
\hline Plasmid ID & Plasmid type & Country $^{a}$ & Isolation Year & Isolation source & SC type ${ }^{b}$ & Between coverage $^{c}(\%)$ & Within coverage $^{d}(\%)$ \\
\hline E0139_2 & $A$ & the Netherlands & 1996 & Non-hospital & 29 & 38 & 85 \\
\hline E0595_2 & $A$ & the Netherlands & 1996 & Pig & 30 & & \\
\hline E0656_2 & $A$ & the Netherlands & 1999 & Non-hospital & 30 & & \\
\hline E6975_4 & B & Greece & 2010 & Hospital & 13 & 25 & 76 \\
\hline E7114_4 & B & Latvia & 2010 & Hospital & 13 & & \\
\hline E7160_5 & B & Slovenia & 2009 & Hospital & 5 & & \\
\hline E7240_4 & B & Greece & 2010 & Hospital & 10 & & \\
\hline E7246_6 & B & Greece & 2009 & Hospital & 13 & & \\
\hline E7471_5 & B & the Netherlands & 2012 & Hospital & 22 & & \\
\hline E8423_3 & B & the Netherlands & 2015 & Hospital & 18 & & \\
\hline E7313_3 & C & the Netherlands & 2012 & Hospital & 2 & 37 & 80 \\
\hline E8014_3 & C & the Netherlands & 2014 & Hospital & 13 & & \\
\hline E8414_4 & C & the Netherlands & 2014 & Hospital & 1 & & \\
\hline E6055_4 & D & Portugal & 2010 & Hospital & 22 & 34 & 73 \\
\hline E8040_4 & D & the Netherlands & 2014 & Hospital & 1 & & \\
\hline E8202_3 & D & the Netherlands & 2015 & Hospital & 1 & & \\
\hline E4227_3 & E & Sweden & 2005 & Chicken & 35 & 29 & 68 \\
\hline E4239_3 & E & Sweden & 2007 & Chicken & 35 & & \\
\hline E6020_3 & $\mathrm{F}$ & Latvia & 2010 & Hospital & 8 & 35 & 67 \\
\hline E6988_5 & $\mathrm{F}$ & Latvia & 2010 & Hospital & 21 & & \\
\hline E7025_5 & $F$ & Latvia & 2010 & Hospital & 23 & & \\
\hline E7040_5 & $\mathrm{F}$ & Latvia & 2010 & Hospital & 21 & & \\
\hline E7067_5 & $\mathrm{F}$ & Latvia & 2010 & Hospital & 21 & & \\
\hline E7070_9 & $\mathrm{F}$ & Latvia & 2010 & Hospital & 21 & & \\
\hline E7207_6 & $\mathrm{F}$ & Greece & 2008 & Hospital & 3 & & \\
\hline
\end{tabular}

${ }^{a}$ Country of sampling isolation

${ }^{b}$ hierBAPS SC. Clonal background of the isolate carrying the plasmid sequence

${ }^{\prime}$ Between coverage refers to the average coverage resulting from pairwise comparisons of complete plasmid sequences belonging to different plasmid types (A-F)

dWithin coverage refers to the average coverage resulting from pairwise comparisons of complete plasmid sequences belonging to the same plasmid type 


\section{Developing a novel plasmid typing scheme based on network clustering}

To establish a partitioning scheme similar to hierBAPS SCs but uniquely based on the similarity between plasmids carrying the vanA-type gene cluster, we first retrieved 26 E. faecium complete vanA plasmids from the same collection of 1644 clinical and non-clinical isolates [21] with known isolation date and country (Table 1). These 26 vanA complete plasmids were previously completed using short- and long-read sequencing data and were previously published in Arredondo-Alonso et al. [21]. To obtain a global picture of the diversity of $v a n A$ plasmids, we included 60 PLSDB plasmid sequences carrying the Tn1546 sequence.

These complete sequences $(n=86)$ were pairwise compared using Mash $(k=21, s=1000)$ and integrated into a network. Based on the k-mer distance distribution (Additional File 2: Fig. S1), we observed two peaks with different heights and resembling a bimodal distribution. Based on this, an optimal cutoff of 0.025 was used to define an edge in the network. This cutoff also split the minor and major modes (peaks) observed in the k-mer distribution (Additional File 2: Fig. S1). This edge cutoff resulted in seven independent subgraphs in the network. For each of the subgraphs, we split them based on their modularity values to retrieve which nodes (plasmid sequences) were highly interconnected and thus signified distinct vanA plasmid types (A-I) with similar content and structure (Fig. 2a). The plasmid types D and E were initially predicted as a single type but were further split based on their average nucleotide coverage (Fig. 2b). Twelve complete plasmid sequences (AP022823.1, E8172_3, NC_005054.1, NC_013317.1, NC_014726.1, NZ_CP012594.1, NZ_CP014531.1, NZ_CP018130.1, NZ_CP019973.1, NZ_CP022486.1, NZ_CP036247.1, NZ_CP040238.1) remained as singletons in the network.

To better understand the modularity and similarity of these plasmid types, we estimated average nucleotide identity (ANI) values using pyani [36]. This allowed retrieving ANI coverage and identity values of the aligned regions between two complete plasmid sequences (pairwise comparisons). We observed that the average coverage between plasmid alignments belonging to the same plasmid type was $79.1 \%$ compared to a coverage of $31.6 \%$ when comparing alignments from different plasmid types. The hierarchical clustering (ward.D2 method) of the alignment coverage reported by pyani showed a high concordance with the plasmid types inferred in our network approach (Fig. 2b). We observed three particular cases of partial disagreement between the plasmid typing and the coverage clustering. These correspond to the plasmid sequences NC_011140.1 (type E), KX810026.1 (type F), and NZ_CP033209.1 (type B) (Fig. $2 \mathrm{~b})$. The position of the nodes representing these sequences in the network approach showed that they were not connected to all members defining the plasmid type. This indicated that these sequences carry k-mer modules not shared in all members of the plasmid type which is also reflected in Fig. 2 b.

We did not observe differences in the average identity values between aligned regions within (98.9\%) and between $(99.7 \%)$ plasmid types indicating a common origin of the plasmid modules present in the different types (Additional File 2: Fig. S2). To exemplify this, we show the plasmid modularity observed in the complete sequences from plasmid type $\mathrm{B}$, since it had the highest $\mathrm{SC}$ diversity and number of associated sequences (Additional File 2: Fig. S3).

In Additional File 3, we provide a detailed genomic characterization of the plasmid types (A-I) with a focus on (i) replication initiator proteins (rep), (ii) Tn1546 variant compared to the original sequence described by Arthur et al. [32], (iii) antimicrobial resistance (AMR) genes distinct from the $v a n A$ gene cluster, and (iv) presence of well-known $E$. faecium plasmid toxin-antitoxin systems such as $\omega-\varepsilon-\zeta$ and axe-txe [47].

\section{Network of predicted plasmid bins}

Next, we performed a de novo plasmid prediction of the sequences carrying the vanA gene cluster in the set of 303 VRE samples with an associated short-read assembly using gplas [30]. This tool uses a combination of machine-learning and a graph-based approach to predict plasmid sequences from short-read graphs [30]. Contigs predicted as belonging to the same plasmid sequence are returned in the same bin.

In 282 isolates (93.1\%), the contig which encodes for the vanA gene cluster (vanA contig) was present in a plasmid bin predicted by gplas. In the remaining isolates ( $n=21,6.9 \%$ ), the $v a n A$ contig remained unbinned and thus we could not predict whether the vanA gene cluster was part of a plasmid. Unbinning of the vanA contig could be caused by a fragmented assembly graph due to, for example, a low sequence coverage. Also, high differences in the k-mer coverage of contigs from the same plasmid can prohibit binning of plasmid contigs with gplas. The inability of gplas to predict the plasmid location of $\operatorname{vanA}$ could also be indicative of a chromosomal location of the $v a n A$ gene cluster. However, manual inspection of the assembly graph in these 21 isolates revealed that the vanA $\mathrm{k}$-mer coverage was clearly higher and distinct from the median k-mer coverage of all contigs, indicative of a plasmid location with a higher copy number compared to the chromosome.

Based on these findings, we concluded that in all 303 vanA VRE isolates with a short-read assembly (100\%), the gene cluster was present in a plasmid background. The preferential presence of the $v a n A$ cluster in a 

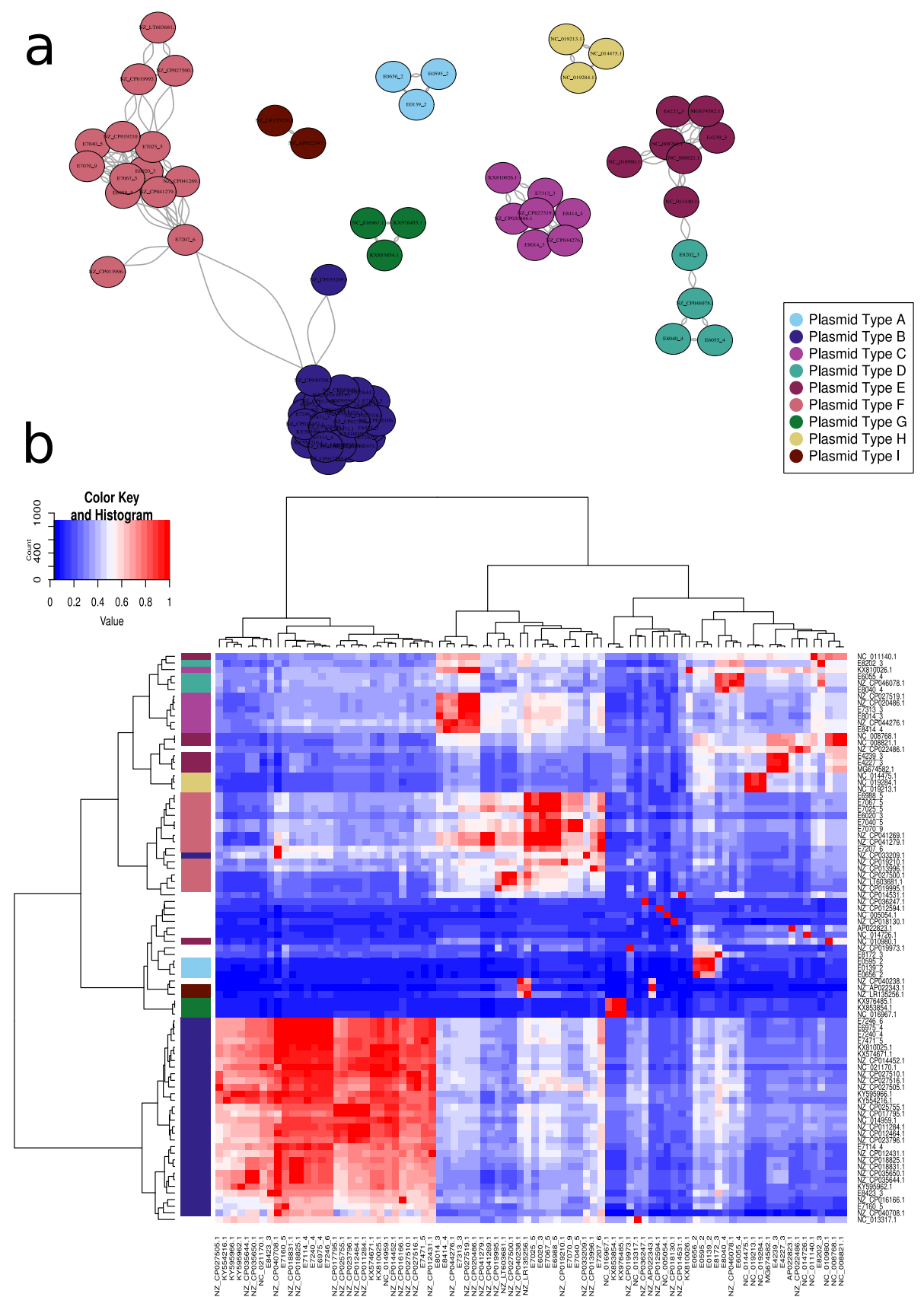

Fig. 2 Definition of the plasmid types (A-I) observed in the 86 retrieved vanA complete plasmid sequences. a Network representation of the vanA complete plasmid sequences based on Mash distances $(k=21, s=1000)$. Nodes $(n=76)$ in the network correspond to complete plasmid sequences and edges were defined if nodes shared a minimum Mash distance of 0.025 . Edges correspond to connections between sequences with a similar k-mer composition. Nodes were colored according to the defined plasmid type assignment. Based on the modularity values and structure of the network components, we identified the plasmid types: A, B, C, D, E, G, H, and I. Twelve complete plasmid sequences (NC_014726.1, AP022823.1, NC_013317.1, NZ_CP012594.1, NC_005054.1, NZ_CP022486.1, NZ_CP014531.1, NZ_CP040238.1, E8172_3,

NZ_CP036247.1, NZ_CP018130.1, NZ_CP019973.1) are not present in the network since they did not share any edges connecting to other nodes (singletons). $\mathbf{b}$ Heatmap and hierarchical clustering (ward.D2 method) of the pyani pairwise alignment coverage obtained from the 86 vanA complete plasmid sequences. On the left side, the plasmid types $(A, B, C, D, E, F, G, H, I)$ previously defined in the network (panel a) are indicated. The twelve plasmid sequences corresponding to singletons are also included in this analysis 
plasmid was previously reported by Freitas et al. (53 isolates, $100 \%$ plasmid encoded vanA) [44] and Wardal et al. (88 isolates, 98\% plasmid encoded vanA) [45]. These 27 isolates in which gplas could not assign the vanA gene cluster to a particular plasmid bin, despite being most likely on a plasmid background were excluded from further analysis.

The 282 isolates containing a predicted gplas plasmid bin were integrated into a network of Mash distances $(k=21, s=1000)$ (Additional File 2: Fig. S4) in which nodes corresponded to predicted plasmid bins and edges to connections of bins with a similar k-mer composition. The distribution of k-mer distances between the plasmid bins (Additional File 2: Fig. S5a) followed the same pattern as observed with the complete plasmid sequences (Additional File 2: Fig. S1). We observed again a bimodal distribution with minor and major modes (Additional File 2: Fig. S5a). The number of resulting components and the average size of the components resulting from different edge thresholds (Additional File 2: Fig. S5b) also indicated that a threshold of 0.025 can be used to draw an edge between nodes in the network shown in
Additional File 2: Fig. S4. The network consisted of 270 nodes with 16 different components ( $>1$ isolate) (Additional File 2: Fig. S4). We observed the presence of a large component (144 nodes) (Additional File 2: Fig. S4, central component) that was split into 3 subgraphs based on its component modularity value (0.42) (Additional File 2: Fig. S4a). In the next analyses, we further focused on components or subgraphs with more than 10 isolates which are indicated in Additional File 2: Fig. S4 and described in Table 2.

\section{Validating the predicted plasmid network by integration of long-read complete plasmid sequences}

To elucidate gene content and synteny of the plasmid bins $(n=282)$, we integrated the complete plasmid sequences (Table 1, Fig. 2) $(n=74)$ used to define the nine plasmid types $\mathrm{A}$ to $\mathrm{I}$, and the singleton sequences $(n=$ 12) with the predicted plasmid bins carrying the vanA gene cluster $(n=282)$. The presence of edges connecting complete plasmids and predicted $v a n A$ plasmid bins revealed that the predictions had a similar k-mer composition and thus further validated the predicted vanA

Table 2 Description of the vanA plasmid bin groups (> 10 isolates) present in the plasmid network (270 nodes) consisting uniquely of predicted plasmid bins. In total, eight distinct plasmid bin groups were defined with a total of 239 isolates

\begin{tabular}{|c|c|c|c|}
\hline vanA plasmid bin group ${ }^{a}$ & Number of isolates & hierBAPS SC & Plasmid type \\
\hline 1 & 11 & SC10 (100\%) & J \\
\hline 2 & 31 & $\begin{array}{l}\text { SC13 }(93.1 \%) \\
\text { SC10 (3.4\%) } \\
\text { SC1 (3.4\%) }\end{array}$ & C \\
\hline 3 & 76 & $\begin{array}{l}\text { SC13 }(72.0 \%) \\
\text { SC2 }(12.0 \%) \\
\text { SC1 }(4.0 \%) \\
\text { SC10 (4.0\%) } \\
\text { SC18 (2.7\%) } \\
\text { SC9 (1.3\%) } \\
\text { SC19 }(1.3 \%) \\
\text { SC22 (1.3\%) } \\
\text { SC23 (1.3\%) }\end{array}$ & C \\
\hline 4 & 62 & $\begin{array}{l}\text { SC17 (66.1\%) } \\
\text { SC10 (17.7\%) } \\
\text { SC18 (12.9\%) } \\
\text { SC22 (1.6\%) } \\
\text { SC23 (1.6\%) }\end{array}$ & B \\
\hline 5 & 20 & $\begin{array}{l}\text { SC13 (50.0\%) } \\
\text { SC17 (40.0\%) } \\
\text { SC7 (5.0\%) } \\
\text { SC8 (5.0\%) }\end{array}$ & I \\
\hline 6 & 17 & $\begin{array}{l}\text { SC20 (47.1\%) } \\
\text { SC18 (35.3\%) } \\
\text { SC1 (11.8\%) } \\
\text { SC2 (5.9\%) }\end{array}$ & $\mathrm{D}$ \\
\hline 7 & 12 & $\begin{array}{l}\text { SC29 }(41.7 \%) \\
\text { SC30 (33.3\%) } \\
\text { SC32 (25.0\%) }\end{array}$ & A \\
\hline 8 & 10 & SC18 (100\%) & E \\
\hline
\end{tabular}

${ }^{a}$ The plasmid bin groups were assigned as carrying a particular plasmid type (A-I) after including the complete vanA plasmid sequences into the network and observing co-clustering of predicted and complete plasmid sequences. For each vanA plasmid bin group predicted plasmid type, we indicated the total number of isolates belonging to the group forming the predicted plasmid type, percentage of SCs, and assigned plasmid type 


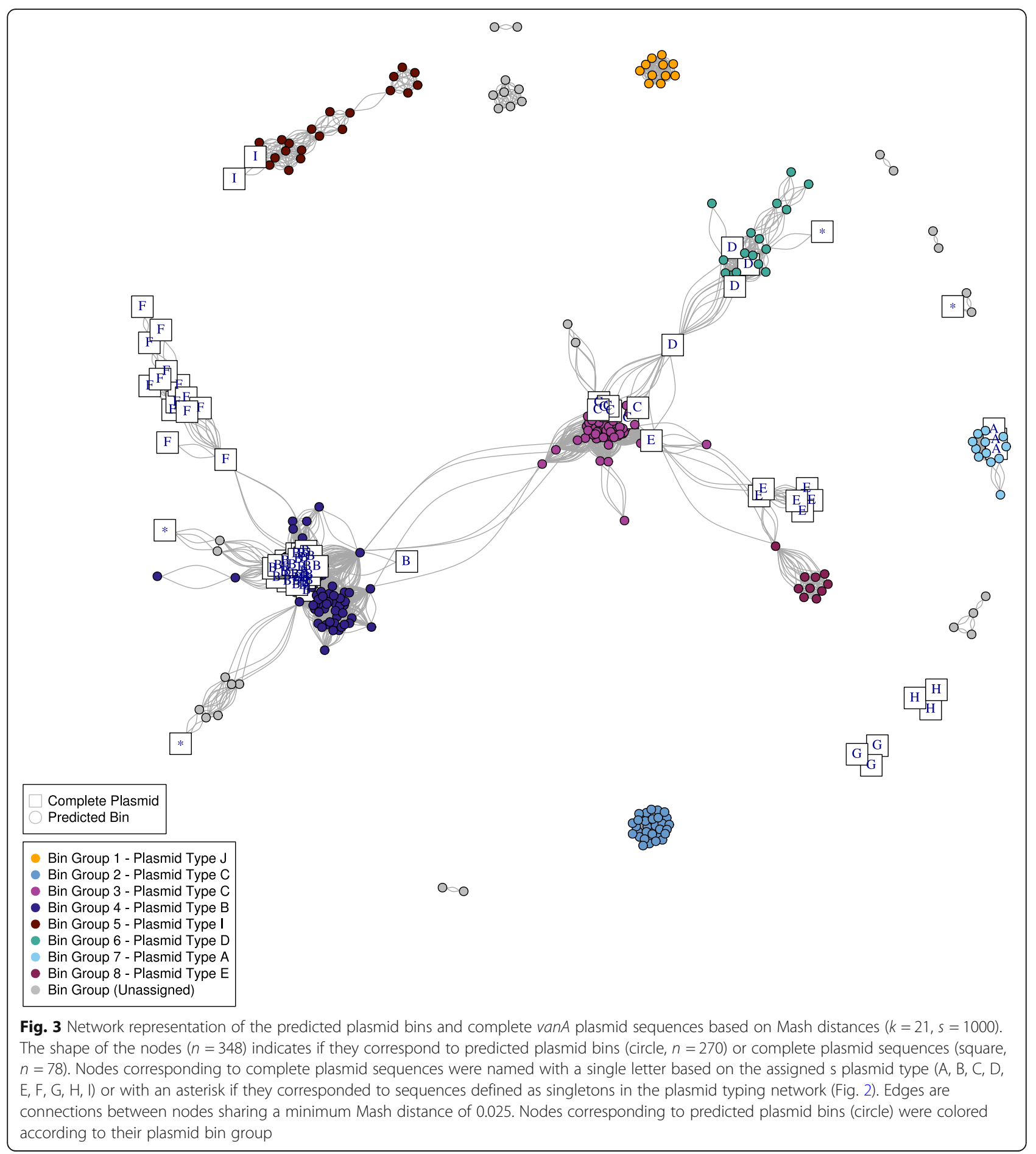

plasmid bins (Additional File 2: Fig. S4). In addition, we included all E. faecium public and complete plasmid sequences carrying the vanA resistance gene cluster derived from the PLSDB database [34]. This resulted in a network of 348 nodes (270 predicted bins, 78 complete plasmid sequences) and 14 components ( $>1$ node) (Fig. 3). We observed that the predicted bin groups 3, 4, 6 , and 7 were co-clustering together with complete plasmid sequences from the plasmid types $\mathrm{C}, \mathrm{B}, \mathrm{D}$, and A respectively (Fig. 3). This indicated that these four bin groups had a k-mer composition highly similar to the plasmid types previously defined. In the predicted bin groups 5 and 8 , we also observed connections between predicted bin groups and complete plasmid sequences. The predicted bin group 5 had connections to two PLSDB plasmid sequences (NZ_AP022343.1, NZ_ 
LR135256.1) belonging to the plasmid type I (Fig. 3). The predicted bin group 8 had a single node connecting to three PLSDB plasmid sequences from the plasmid type E (NC_008768.1, NC_008821.1, NC_011140.1) (Fig. $3)$. However, in these two bin groups $(5,8)$ the number of bins connecting to plasmid sequences was lower than for the previous bin groups $(3,4,6,7)$ which indicated a more heterogeneous composition of the k-mer content for these two plasmid bin groups.

The inclusion of plasmids completed with long-read sequencing data was fundamental to deduce that the plasmid prediction of the isolates from bin group 2 was incorrect. In this bin, one of the isolates (E8014) had an associated complete plasmid sequence (E8014_3, Fig. 2) but we observed no connections between its predicted gplas bin, present at plasmid bin 2, and its associated plasmid sequence (E8014_3, plasmid type C). Based on this, we concluded that the prediction given by gplas was erroneous and the k-mer composition of plasmid bin group 2 should be similar to the plasmid type $\mathrm{C}$ despite being disconnected in the network. We only considered the group bin 1 as carrying a novel plasmid type (J) since it was not connected to any complete plasmid sequence (Fig. 3).

This network approach allowed assigning 239 isolates with a particular plasmid type (A, B, C, D, E, I, J) (Table 2). In Additional File 3 , we provide an extensive characterization of the plasmid bin groups observed in the network (Fig. 3) based on group diversity regarding SC, Tn1546 variants, geographical site, and year of isolation.

For the following analyses, we considered all isolates $(n=225)$ for which we had complete metadata information (hospital and isolation date), SC, plasmid type, Tn1546 assignments, and were present in the PopPUNK core-genome tree.

SC, plasmid type, and Tn1546 assignments and the nomenclature used to name these transposon variants are outlined in Table 3 and Additional File 4: Table S2.

In Fig. 4, we combined the core-genome-based neighbor-joining tree with SC, vanA plasmid type, and Tn1546 assignments. Some closely related isolates in the core-genome tree had distinct predicted vanA plasmid types as exemplified by $\mathrm{SC} 17$ containing plasmid types B and I (Fig. 4). However, these plasmid types contained the same Tn1546 variant (46MN), which is characterized by deletions of orf1 and orf2 and SNPs T7658C, G8234T. This observation suggested that plasmid types $\mathrm{B}$ and I co-resided in the same isolates and that Tn1546 was internally mobilized between these two plasmid types within the same SC. The isolates belonging to the clonal background SC10 were also closely related in the core-genome tree but shared distinct plasmid types and were associated to different $\mathrm{Tn} 1546$ variants: (i) plasmid
Table 3 Ad hoc scheme used to name the Tn1546 variants present in the predicted vanA plasmid sequences

\begin{tabular}{lll}
\hline Type & Position & Nomenclature \\
\hline SNP & C806T & 1 \\
SNP & G3966T & 2 \\
SNP & G4351T & 3 \\
SNP & T7658C & 4 \\
SNP & G7747T & 5 \\
SNP & G8234T & 6 \\
SNP & C8833T & 7 \\
SNP & C9692T & 8 \\
Deletion & orf1 & M \\
Deletion & orf2 & $\mathrm{N}$ \\
Deletion & vanR & $\mathrm{R}$ \\
Deletion & vanS & $\mathrm{S}$ \\
Deletion & vanH & $\mathrm{H}$ \\
Deletion & vanA & $\mathrm{A}$ \\
Deletion & vanX & $\mathrm{X}$ \\
Deletion & vanY & $\mathrm{Y}$ \\
Deletion & vanZ & $\mathrm{Z}$ \\
Deletion & Intergenic & $\mathrm{I}$ \\
\hline
\end{tabular}

type J with 46 variant (SNPs G7747T+C8833T); (ii) plasmid type B associated mostly to $46 \mathrm{M}, 46 \mathrm{MN}$, and 468MN; (iii) plasmid type $\mathrm{C}$ with MNI variant (deletions 1-3417 and 8650-8827). This indicated that isolates from the clonal background SC10 have horizontally acquired different vanA plasmids associated with distinct Tn1546 variants. This situation is also apparent for SC18 isolates which were closely related in the core-genome tree but carried three distinct plasmid types (B, D, E) which were associated with distinct Tn1546 variants (Fig. 4).

However, we observed overall a high concordance between the SC and Tn1546 assignments (Fig. 4), which suggested that clonal spread was the main driver of the $v a n A$ resistance gene cluster dissemination. To facilitate the exploration of the results, SC, plasmid type, and Tn1546 assignments were also integrated into the Microreact project https://microreact.org/project/ FCUD_d1zt

\section{Dynamics of vanA-type resistance dissemination}

The identification of the seven vanA plasmid types (A, $\mathrm{B}, \mathrm{C}, \mathrm{D}, \mathrm{E} \mathrm{I}, \mathrm{J})$ present in our Dutch VRE collection allowed us to estimate the contribution of the nested genetic elements, clone (defined as hierBAPS-based SC), plasmid type, and $\mathrm{Tn} 1546$ variant, into the dissemination of the vanA gene cluster.

To establish this, we first grouped VRE isolates $(n=$ 225) with a potential epidemiological link which corresponded to isolates sampled from two consecutive years. 


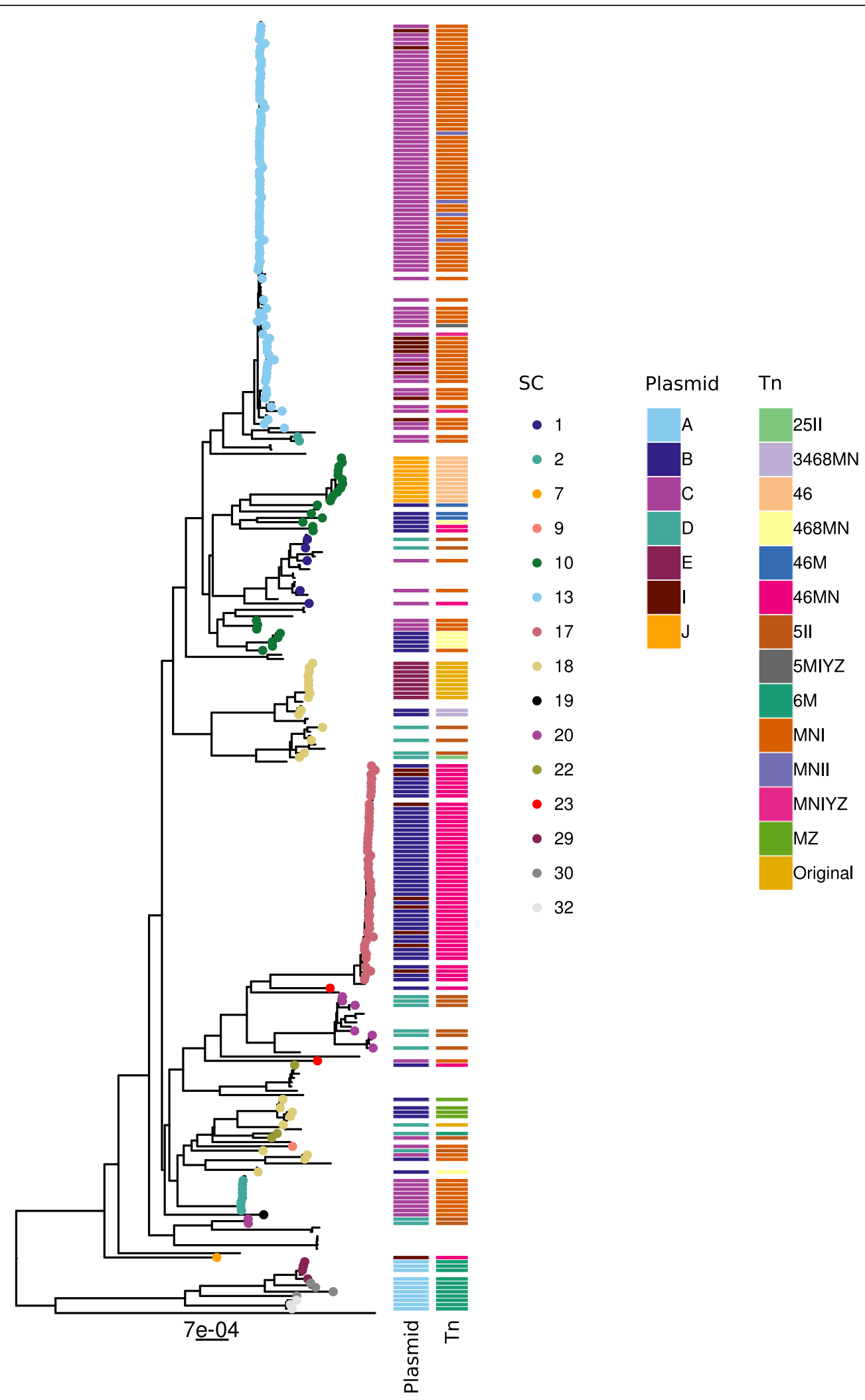

Fig. 4 (See legend on next page.) 
(See figure on previous page.)

Fig. 4 Core-genome-based tree generated by PopPUNK in 303 VRE isolates with an associated short-read assembly. For 225 isolates, we had complete metadata information (hospital and isolation date), hierBAPS SC, plasmid type, and Tn1546 assignments. The nodes of the core-genome tree corresponding to this subset of 225 isolates are colored based on their associated hierBAPS SC $(n=15)$. The plasmid type $(n=6)$ and Tn1546 variants $(n=14)$ assignments are plotted in two distinct and adjacent panels. Tn1546 variants were named following the ad hoc scheme shown in Table 3. This information can also be fully inspected interactively in the following Microreact project: https://microreact.org/project/FCUD_d1zt

From each year interval, we considered all windows of 12 consecutive months with at least 10 isolates to estimate the dissemination of vanA resistance. This approach was undertaken to avoid grouping the isolates based uniquely on their isolation year which neglects the comparison between isolates recovered within a few months. For each window, we performed a pairwise "all vs. all" comparison to define the most likely scenarios of the dissemination of the vanA gene cluster (see the section "Contribution of nested genomic elements in the dissemination of vanA-type gene cluster" in the "Methods"). We performed the same analysis on Dutch regions (Additional File 2: Fig. S6) and hospitals (Additional File 2: Fig. S7) to identify particular SC, plasmid type, and $\operatorname{Tn} 1546$ variants involved in the dissemination at particular time periods.

On a country-wide level, we observed that most of the isolates did not share the same $\mathrm{Tn} 1546$ variant and thus were categorized as unrelated (avg. freq $=0.59$ ) (Fig. 5). Between isolates with an identical Tn1546 variant, clonal spread defined by observing the same SC, plasmid type, and $\operatorname{Tn} 1546$ was the predominant mode of vancomycin resistance spread (avg. freq $=0.27$ ) and clearly dominated the dissemination of vanA between January 2013 and December 2014 (freq $=0.44)$ (Fig. 5). This clonal spread was accentuated in some regions like Flevoland (January 2012-December 2013) (freq $=0.70$ ) or North-Holland (January 2012-December 2013) (freq $=0.75$ ) in which the clone SC17, associated with plasmid type B and Tn1546 variant 46MN (deletions in orf1 and orf2 13343, SNPs T7658C, G8234T), was responsible for the spread of vancomycin resistance (Additional File 2: Fig. S6). In this last region (North-Holland), the dissemination of the vanA cluster was still mainly dominated by clonal spread between January 2013 and December 2014 but was driven by a distinct clone, SC13, associated with the plasmid type $\mathrm{C}$ and the Tn1546 variant MNI (deletions in orf1 and orf2 1-3343 and the intergenic region 8650-8827) (Additional File 2: Fig. S6). We also quantified events of clonal spread (avg. freq $=0.05$ ) (Fig. 5, category "Clonal SC + Transposon") in which isolates shared identical SC and Tn1546 variants but carried a distinct plasmid type. These cases most likely correspond to instances of Tn1546 mobilization between plasmids co-existing in the same isolates as observed in Overijssel (January 2012-December 2013) (freq $=0.32$ ) in which SC17 and the Tn1546 46MN variant (deletions in orf1, orf2 1-3343, SNPs T7658C, G8234T) were found mostly in the plasmid types B and I. Overall, clonal spread either by observing the entire nested genomic system or the same SC and Tn1546 variant had a frequency of $\sim 0.32$.

Plasmid dissemination characterized by the same plasmid type and Tn1546 variant but distinct SC (HGT) had an average frequency of 0.07 (Fig. 5). During January 2014 and December 2015, we observed an increase in the frequency of plasmid spread (0.14) and more accentuated in the region of South-Holland (January 2014December 2015) (Additional File 2: Fig. S6). In this region, we identified four distinct clonal backgrounds (SCs $2,10,13,19)$ carrying the same plasmid type $C$ with the Tn1546 MNI variant (deletions in orf1 and orf2 1-3417 and intergenic region 8650-8827).

Finally, we also observed cases of isolates sharing an identical Tn1546 variant (avg. freq $=0.02$ ) (Fig. 5, category "Mixed Transposon") on distinct clonal backgrounds and plasmid types. This could be the result of mobilization of $\operatorname{Tn} 1546$ between plasmids from the same $\mathrm{SC}$ and posterior plasmid transfer of the vanA gene cluster to a distinct SC.

\section{Discussion}

In this study, we propose a graph-based plasmid prediction coupled with a network analysis of the shared plasmid k-mer content to elucidate the dynamics of vertical and horizontal dissemination of AMR genes with shortread WGS. We used this approach to elucidate the mode of vanA-type vancomycin resistance dissemination. Clonal and horizontal mobilization of vanA were previously documented but the quantity of these events on a large scale is largely unexplored. Our analysis permitted us to distinguish and quantify dissemination occurring by (i) clonal spread of the entire nested system (identical SC, plasmid, Tn1546), (ii) plasmid dissemination, (iii) clonal dissemination associated to Tn1546 mobilization (identical SC, Tn1546), and (iv) mixed events of clonal and plasmid dissemination resulting in $\operatorname{Tn} 1546$ mobilization (same transposon variant, distinct SC and plasmid types). This revealed that clonal dissemination was the predominant $(\sim 32 \%)$ mode of spread of vanA type of vancomycin followed by plasmid dissemination $(\sim 7 \%)$ and mixed dissemination of Tn1546 involving different SC and plasmid types ( 2\%). However, we also observed that most of the cases were unrelated ( 59\%) 


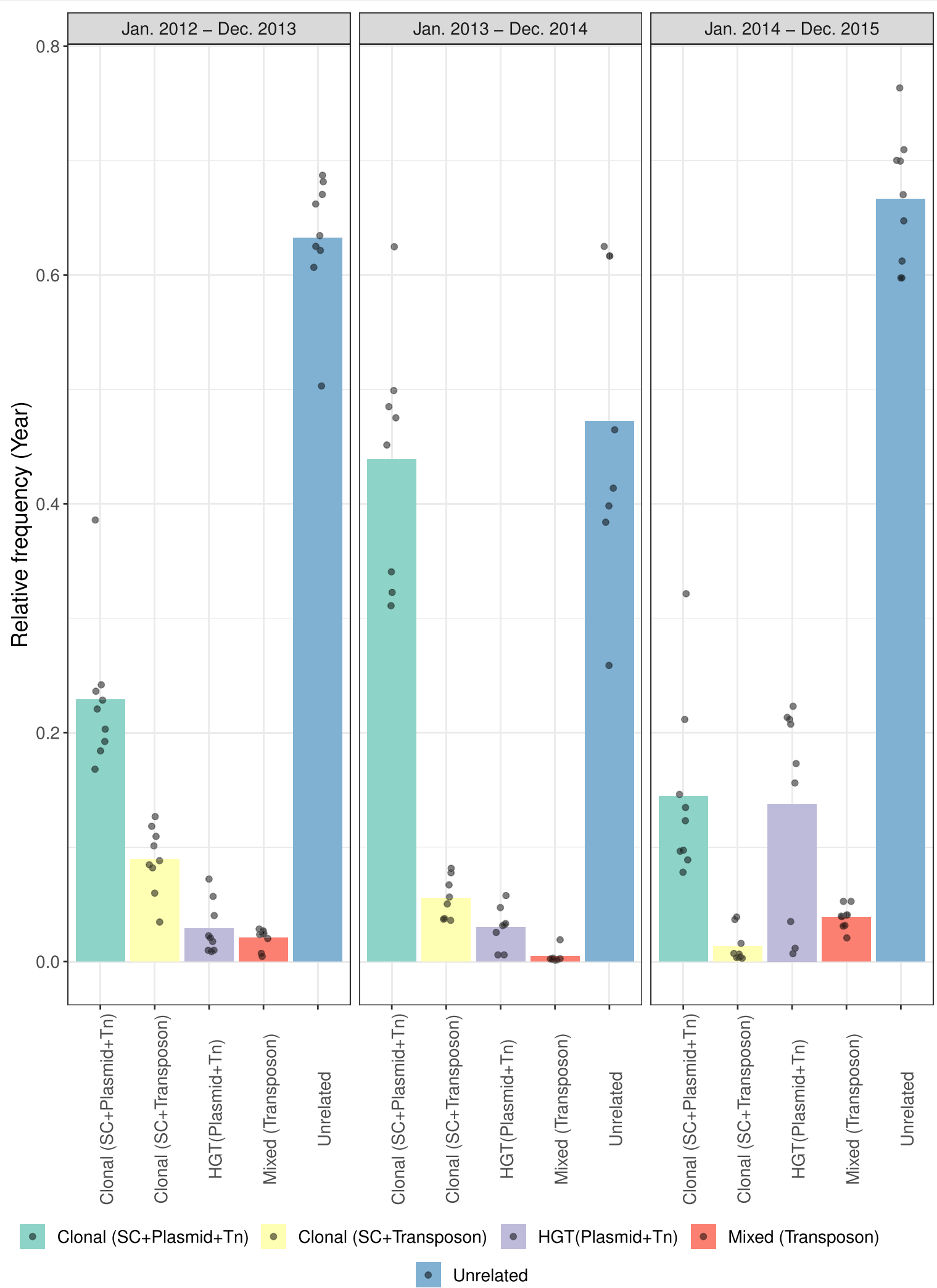

Fig. 5 (See legend on next page.) 
(See figure on previous page.)

Fig. 5 Country-wide contribution of dissemination modes in the spread of vanA-type vancomycin resistance in the Netherlands. VRE isolates ( $n=$ 225) with complete metadata, hierBAPS SC, plasmid type, and Tn1546 assignments were considered. In this analysis, time intervals of 2 years (January 2012-December 2013; January 2013-December 2014; January 2014-December 2015) were considered to group the isolates. For each time interval, all windows of 12 consecutive months with at least 10 isolates were considered. From each window, we undertook a pairwise comparison to estimate the frequency of the following events: (i) clonal dissemination, pairs of isolates sharing the same hierBAPS SC, vanA plasmid type, and Tn1546 variant; (ii) plasmid dissemination, pairs of isolates sharing the same vanA plasmid type and Tn1546 variant but distinct hierBAPS SC; (iii) clonal dissemination associated with Tn1546 mobilization, pairs of isolates sharing the same hierBAPS SC and Tn1546 variant but different vanA plasmid type; (iv) Tn1546 mobilization linked to clonal and horizontal dissemination, pairs of isolates sharing the same Tn1546 variant and distinct hierBAPS SC and vanA plasmid type; and (v) unrelated cases, pairs of isolates with distinct Tn1546 variants

which may indicate distinct and multiple introductions of vancomycin resistance in the Netherlands during 2012 and 2015. The approach presented here can be applied to study clonal and HGT dissemination of other AMR genes, such as carbapanamese genes $\left(b l a_{\mathrm{kpc}}\right)$ in Enterobacteriaceae or colistin resistance genes $(\mathrm{mcr})$ in Escherichia coli.

Previous studies have described the importance of both clonal and horizontal Tn1546 dissemination in the emergence of VRE isolates $[18,20]$. However, a quantitative assessment of the contribution from the different nested genomic elements in the dissemination of vancomycin resistance has not been previously performed. Combining existing short-read WGS with complete vanA plasmids allowed us to define and characterize several plasmid types present in the collection. The integration of previously completed $v a n A$ plasmids was essential to elucidate the genetic content of the vanA plasmid bins present in our predicted network. These vanA plasmid types were defined by $\sim 99 \%$ identity and $\sim 79 \%$ coverage and were present in different clonal backgrounds (SCs) and carried a predominant Tn1546 variant that accumulated additional SNPs and/or deletions (Additional File 3). The genomic relatedness of strains, using hierBAPS, plasmid type and Tn1546 variant calling was combined to sketch a comprehensive picture of the molecular epidemiology of vanA-type vancomycin resistance in Dutch hospitals.

Transposition of Tn1546 between different plasmids has been reported before. Heaton et al. showed the transfer of the Tn1546 element from a nonconjugative to a conjugative plasmid in the same bacterial cell which was mediated by flanking IS1216 elements [19]. This event can result in the presence of an identical $\mathrm{Tn} 1546$ variant in distinct plasmid types but still being carried and spread in the same clonal background (SC). Furthermore, horizontal dissemination by larger units than the $\mathrm{Tn} 1546$ as part of a composite transposon between co-existing plasmids has also been previously documented $[18,20]$. Moreover, this observation could also explain the mosaicism observed in the plasmidome of hospitalized patients in which plasmid blocks are exchanged between different plasmid types [48].
The dissemination of the vanA gene cluster can also occur at a plasmid level in which both plasmid type and Tn1546 variant are horizontally disseminated, as observed in South-Holland between 2014 and 2015. This type of HGT dissemination can be driven by conjugative plasmids but also from non-conjugative mobilizable plasmids. The presence of non-conjugative plasmids coresiding with conjugative plasmids in the same cell can enhance the horizontal dissemination of both plasmid sequences. This observation has been experimentally validated for the E. faecium pHT $\beta$-like plasmid, which contained an efficient conjugation machinery, and allowed the mobilization of other multi-drug resistant and nonconjugative plasmids present in the same cell [49].

The dissemination of vancomycin resistance was previously investigated using WGS in several recent studies [11, 50-53] but seldom with a focus on distinguishing clonal and plasmid outbreaks. One exception is the study by Pinholt et al. [14] that used a combination of short-read and long-read sequencing to describe the clonal expansion of VRE in the Capital Region of Denmark between 2012 and 2015. Here, ST80 was defined as responsible for the first observed local outbreaks. These clonal isolates subsequently spread to other hospitals in the same region. The plasmid bearing the $v a n A$ gene cluster was disseminated to other, nonclonally related vancomycin-susceptible isolates. In our data set covering the same time frame, ST80 represented by SC18 was also a predominant clone (Fig. 1a).

The emergence of $v a n A$-type resistance was also investigated in Australia during 2015 using a combination of short- and long-read sequencing [15]. The study showed the presence of several vanA plasmid types which were dominant in each ST group with distinct Tn1546 variants. This unraveled that, in Australia, the emergence of the vanA-type resistance most likely occurred by multiple introductions of different clones which suggested that HGT is not solely responsible for the spread of the vanA gene cluster, which is in line with our own results.

Both these studies [14, 15], however, followed a reference-based approach to deduce the presence of a particular vanA plasmid type. This could mask the presence of plasmid types which are distinct from the selected reference plasmid(s). In our study, predicted 
plasmids have been integrated into a network that avoids the arbitrary usage of a reference plasmid and takes plasmid modularity into account. Bipartite networks were previously postulated to explore the pangenome of bacterial species with a particular emphasis on the accessory genome [54]. A network approach also allows classifying plasmids in the absence of common evolutionary history as it can integrate both horizontal and vertical inheritance, in contrast to phylogenetic trees $[55,56]$. The classification of plasmids based on k-mer similarity networks has also recently been proposed by Acman et al. [57].

A focus on the core genome can overestimate the number of isolates that are considered as non-related and thus missing potential epidemiological links. In line with Harris et al. [58], we encourage the shift from a traditional core-genome view on outbreak investigations to a new perspective that also includes the analyses of HGT mobilization of AMR genes to effectively confirm potential epidemiological links and correctly evaluate the effectiveness of infection control policies. We showed that highly similar plasmids can be transferred between different SC's which challenges the interpretation of AMR outbreak studies that are solely focused on coregenome analysis. A factor that contributes to this clonality centricity is the limitations inherent to short-read WGS from which the assembly of plasmids is difficult and error-prone due to the high number of repeated sequences [59]. For full resolution, many studies recommend long-read sequencing to complete chromosomes and plasmids $[16,59,60]$. We show in this study, however, that distinguishing between different modes of spread is feasible also in the absence of long-read data.

A limitation of this work is that only a single colony per isolate was sequenced. This masked the true underlying diversity present in the bacterial population and could have prohibited the detection of potential epidemiological links. While our data set allowed us to define the most likely events of dissemination, we lacked patient admission and ward movement information to discern transmission routes and events, as previously shown by Raven et al. [11] and Neumann et al. [61]. The absence of data on vancomycin-susceptible isolates also prevented us to deduce how the distinct plasmid types and $\operatorname{Tn} 1546$ variants were introduced into the Dutch hospitals. Nonetheless, we succeeded to provide one of the first quantitative assessments to discern the dynamics and contribution of clonal and horizontal transmission in the dissemination of vancomycin resistance.

\section{Conclusions}

This study has shown that in VRE-related cases, clonal dissemination was the preferential mode of dissemination of vanA-type vancomycin resistance observed in
Dutch hospitals between 2012 and 2015. However, we also detected outbreak settings in which HGT plasmid dissemination contributed most to the spread of resistance. Our analyses showed the importance of taking all nested genomic elements into account to effectively elucidate how resistance spreads in healthcare settings. This is fundamental to corroborate potential epidemiological links that could be neglected by uniquely considering strain relatedness. Only then, the effectiveness of current infection control policies to prevent AMR spread can be truly assessed.

\section{Supplementary Information}

The online version contains supplementary material available at https://doi. org/10.1186/s13073-020-00825-3.

Additional file 1: Table S1. Metadata table related to the $309 \mathrm{vanA}$ type vancomycin-resistant Enterococcus faecium isolates. (CSV 20 kb)

Additional file 2. Supplementary Figures: Fig. S1-Fig. S8.

Additional file 3. Supplementary Results: A) Characterization of the sequences defined plasmid types $(A-I)$. B) Characterization of the predicted vanA plasmid groups $(1-8,>10$ isolates) identified in the network of predicted plasmid bins.

Additional file 4: Table S2. Genome assignments and metadata information of the $225 \mathrm{VRE}$ isolates considered to estimate the most likely events of vanA dissemination. (CSV $16 \mathrm{~kb}$ )

\section{Abbreviations}

WHO: World Health Organization; VRE: Vancomycin-resistant Enterococcus faecium; WGS: Whole-genome sequencing; HGT: Horizontal gene transfer; AMR: Antimicrobial resistance; SC: Sequencing cluster; SNP: Single nucleotide polymorphism; ORF: Open reading frame

\section{Acknowledgements}

We would like to acknowledge the following Dutch hospitals for providing us with the 309 VRE isolates included in this study: Admiraal de Ruyter Goes, Albert Schweitzer Dordrecht, Amphia ziekenhuis Breda, Amsterdam UMC location AMC, St. Antonius location Nieuwegein, St. Antonius location Oudenrijn Utrecht, AZM Maastricht, Diakonessenhuis Utrecht, Dijklander ziekenhuis Hoorn, Erasmus MC Rotterdam, Flevoziekenhuis Almere, Gelderse Vallei Ede, Isala klinieken Zwolle, Laurentius Ziekenhuis Roermond, Leyenburg Den Haag, LUMC Leiden, Maasstad Rotterdam, MC Alkmaar, Meander Medisch Centrum Amersfoort, MST Enschede, OLVG location East Amsterdam, OLVG location West Amsterdam, Rijnstate Arnhem, Spaarne Haarlem, St Jansdal Harderwijk, St. Antonius Woerden, UMC Utrecht, UMCG Groningen, Viecuri MC Venlo, Westeinde Den Haag, Zuyderland MC (previously Atrium Heerlen and Orbis Sittard).

\section{Authors' contributions}

A.C.S and R.J.L.W initiated and supervised the project. S.A-A performed the computational analysis. SA-A, A.C.S, and R.J.L.W wrote the manuscript. All authors read and commented on the manuscript. All authors read and approved the final manuscript.

\section{Funding}

This work was supported by the Joint Programming Initiative in Antimicrobial Resistance [JPIAMR Third call, STARCS, JPIAMR2016-AC16/00039 to S.A.-A. and R.J.L.W.]. The study was also partially funded by ZonMW [The Netherlands, project number 541003005 to A.C.S] and by the European Research Council [grant number 742158 to J.C.]. 
(A-F) are also available through the European Nucleotide project PRJEB28495 https://www.ebi.ac.uk/ena/browser/view/PRJEB28495 [62] and the gitlab project https://gitlab.com/sirarredondo/vancomycin_dissemination [43]. The plasmid sequences derived from the PLSDB database [34] considered in the manuscript have the following European Nucleotide Archive number accessions: AP022823.1, KX574671.1,KX810025.1,KX810026.1, KX853854.1, KX976485.1, KY554216.1, KY595962.1, KY595966.1, MG674582.1, NC_005054.1, NC_008768.1, NC_008821.1, NC_010980.1, NC_011140.1, NC_013317.1, NC_014475.1, NC_014726.1, NC_014959.1, NC_016967.1, NC_019213.1, NC_019284.1, NC_021170.1, NZ_AP022343.1, NZ_CP011284.1, NZ_CP012431.1, NZ_CP012464.1, NZ_CP012594.1, NZ_CP013996.1, NZ_CP014452.1, NZ_CP014531.1, NZ_CP016166.1, NZ_CP017795.1, NZ_CP018130.1, NZ_CP018825.1, NZ_CP018831.1, NZ_CP019210.1, NZ_CP019973.1, NZ_CP019995.1, NZ_CP020486.1, NZ_CP022486.1, NZ_CP023796.1, NZ_CP025755.1, NZ_CP027500.1, NZ_CP027505.1, NZ_CP027510.1, NZ_CP027516.1, NZ_CP027519.1, NZ_CP033209.1, NZ_CP035644.1, NZ_CP035650.1, NZ_CP036247.1, NZ_CP040238.1, NZ_CP040708.1, NZ_CP041269.1, NZ_CP041279.1 NZ_CP044276.1 NZ_CP046078.1, and NZLR135256.1, NZ_LT603681.1. These PLSDB sequences are also available at https://gitlab.com/sirarredondo/vancomycin_ dissemination [43]

The complete code used to generate the results present in this manuscript is provided in a RMarkdown document available through https://gitlab.com/ sirarredondo/vancomycin_dissemination [43].

\section{Ethics approval and consent to participate}

Not applicable.

\section{Consent for publication}

Not applicable.

\section{Competing interests}

The authors declare that they have no competing interests.

\begin{abstract}
Author details
${ }^{1}$ Department of Medical Microbiology, University Medical Center Utrecht, Utrecht, The Netherlands. 'Department of Biostatistics, University of Oslo, Oslo, Norway. ${ }^{3}$ Pathogen Genomics, Wellcome Trust Sanger Institute, Cambridge CB10 1SA, UK. ${ }^{4}$ Department of Mathematics and Statistics, Helsinki Institute of Information Technology (HIIT), Fl-00014 University of Helsinki, Helsinki, Finland.
\end{abstract}

Received: 6 July 2020 Accepted: 30 December 2020

Published online: 20 January 2021

\section{References}

1. Weiner-Lastinger LM, Abner S, Edwards JR, Kallen AJ, Karlsson M, Magill SS, et al. Antimicrobial-resistant pathogens associated with adult healthcareassociated infections: summary of data reported to the National Healthcare Safety Network, 2015-2017. Infect Control Hosp Epidemiol. 2020;41:1-18.

2. Novosad SA, Fike L, Dudeck MA, Allen-Bridson K, Edwards JR, Edens C, et al. Pathogens causing central-line-associated bloodstream infections in acutecare hospitals-United States, 2011-2017. Infect Control Hosp Epidemiol. 2020;41:313-9.

3. Tacconelli E, Magrini N, Kahlmeter G, Singh N. Global priority list of antibiotic-resistant bacteria to guide research, discovery, and development of new antibiotics. World Health Organization. 2017;27:1-7.

4. Cattoir V, Leclercq R. Twenty-five years of shared life with vancomycinresistant enterococci: is it time to divorce? J Antimicrob Chemother. 2013; 68:731-42.

5. Lebreton F, Depardieu F, Bourdon N, Fines-Guyon M, Berger P, Camiade S, et al. D-Ala-d-Ser VanN-type transferable vancomycin resistance in Enterococcus faecium. Antimicrob Agents Chemother. 2011;55:4606-12.

6. Xu X, Lin D, Yan G, Ye X, Wu S, Guo Y, et al. vanM, a new glycopeptide resistance gene cluster found in Enterococcus faecium. Antimicrob Agents Chemother. 2010;54:4643-7.

7. Guzman Prieto AM, van Schaik W, Rogers MRC, Coque TM, Baquero F, Corander J, et al. Global emergence and dissemination of enterococci as nosocomial pathogens: attack of the clones? Front Microbiol. 2016;7:788.
8. Saeedi B, Hallgren A, Jonasson J, Nilsson LE, Hanberger H, Isaksson B. Modified pulsed-field gel electrophoresis protocol for typing of enterococci. APMIS. 2002;110:869-74.

9. Top J, Schouls LM, Bonten MJM, Willems RJL. Multiple-locus variablenumber tandem repeat analysis, a novel typing scheme to study the genetic relatedness and epidemiology of Enterococcus faecium isolates. J Clin Microbiol. 2004;42:4503-11.

10. Homan WL, Tribe D, Poznanski S, Li M, Hogg G, Spalburg E, et al. Multilocus sequence typing scheme for Enterococcus faecium. J Clin Microbiol. 2002;40: 1963-71.

11. Raven KE, Gouliouris T, Brodrick H, Coll F, Brown NM, Reynolds R, et al. Complex routes of nosocomial vancomycin-resistant Enterococcus faecium transmission revealed by genome sequencing. Clin Infect Dis. 2017;64:886-93.

12. Werner G, Freitas AR, Coque TM, Sollid JE, Lester C, Hammerum AM, et al. Host range of enterococcal vanA plasmids among Gram-positive intestinal bacteria. J Antimicrob Chemother. 2011;66:273-82.

13. Freitas AR, Coque TM, Novais C, Hammerum AM, Lester CH, Zervos MJ, et al. Human and swine hosts share vancomycin-resistant Enterococcus faecium CC17 and CC5 and Enterococcus faecalis CC2 clonal clusters harboring Tn1546 on indistinguishable plasmids. J Clin Microbiol. 2011;49:925-31.

14. Pinholt M, Bayliss SC, Gumpert $H$, Worning $P$, Jensen WS, Pedersen $M$, et al. WGS of 1058 Enterococcus faecium from Copenhagen, Denmark, reveals rapid clonal expansion of vancomycin-resistant clone ST80 combined with widespread dissemination of a vanA-containing plasmid and acquisition of a heterogeneous accessory genome. J Antimicrob Chemother. 2019;74: 1776-85.

15. Lee RS, Gonçalves da Silva A, Baines SL, Strachan J, Ballard S, Carter GP, et al. The changing landscape of vancomycin-resistant Enterococcus faecium in Australia: a population-level genomic study. J Antimicrob Chemother. 2018; 73:3268-78.

16. Sheppard AE, Stoesser N, Wilson DJ, Sebra R, Kasarskis A, Anson LW, et al. Nested Russian doll-like genetic mobility drives rapid dissemination of the carbapenem resistance gene blakPC. Antimicrob Agents Chemother. 2016;60:3767-78.

17. Freitas AR, Novais C, Tedim AP, Francia MV, Baquero F, Peixe L, et al. Microevolutionary events involving narrow host plasmids influences local fixation of vancomycin-resistance in Enterococcus populations. PLoS One. 2013;8:e60589.

18. Novais C, Freitas AR, Sousa JC, Baquero F, Coque TM, Peixe LV. Diversity of Tn1546 and its role in the dissemination of vancomycin-resistant enterococci in Portugal. Antimicrob Agents Chemother. 2008;52:1001-8.

19. Heaton MP, Discotto LF, Pucci MJ, Handwerger S. Mobilization of vancomycin resistance by transposon-mediated fusion of a VanA plasmid with an Enterococcus faecium sex pheromone-response plasmid. Gene. 1996;171:9-17.

20. Sletvold H, Johnsen PJ, Wikmark O-G, Simonsen GS, Sundsfjord A, Nielsen KM. Tn1546 is part of a larger plasmid-encoded genetic unit horizontally disseminated among clonal Enterococcus faecium lineages. J Antimicrob Chemother. 2010;65:1894-906.

21. Arredondo-Alonso S, Top J, McNally A, Puranen S, Pesonen M, Pensar J, et al. Plasmids shaped the recent emergence of the major nosocomial pathogen Enterococcus faecium. MBio. 2020;11 Available from: https://doi. org/10.1128/mBio.03284-19.

22. Arredondo-Alonso S, Rogers MRC, Braat JC, Verschuuren TD, Top J, Corander J, et al. mlplasmids: a user-friendly tool to predict plasmid- and chromosome-derived sequences for single species. Microb Genom. 2018; Available from: https://doi.org/10.1099/mgen.0.000224

23. Krueger F. Trim Galore: a wrapper tool around Cutadapt and FastQC to consistently apply quality and adapter trimming to FastQ files, with some extra functionality for Mspl-digested RRBS-type (reduced representation Bisufite-Seq) libraries. 2012. Available from: https://www.bioinformatics. babraham.ac.uk/projects/trim_galore/

24. Martin M. Cutadapt removes adapter sequences from high-throughput sequencing reads. EMBnet.journal. 2011;17:10-2.

25. Andrews S, Others. FastQC: a quality control tool for high throughput sequence data. Babraham Bioinformatics, Babraham Institute, Cambridge, United Kingdom; 2010.

26. Wick RR, Judd LM, Gorrie CL, Holt KE. Unicycler: resolving bacterial genome assemblies from short and long sequencing reads. PLoS Comput Biol. 2017;13: e1005595.

27. Bankevich A, Nurk S, Antipov D, Gurevich AA, Dvorkin M, Kulikov AS, et al. SPAdes: a new genome assembly algorithm and its applications to singlecell sequencing. J Comput Biol. 2012;19:455-77. 
28. Zankari E, Hasman $H$, Cosentino S, Vestergaard M, Rasmussen S, Lund O, et al. Identification of acquired antimicrobial resistance genes. J Antimicrob Chemother. 2012;67:2640-4.

29. Lees JA, Harris SR, Tonkin-Hill G, Gladstone RA, Lo SW, Weiser JN, et al. Fast and flexible bacterial genomic epidemiology with PopPUNK. Genome Res. 2019;29:304-16.

30. Arredondo-Alonso S, Bootsma M, Hein Y, Rogers MRC, Corander J, Willems RJL, et al. gplas: a comprehensive tool for plasmid analysis using short-read graphs. Bioinformatics. 2020; Available from: https://doi.org/10.1093/ bioinformatics/btaa233

31. Sheppard AE, Stoesser N, German-Mesner I, Vegesana K, Sarah Walker A, Crook DW, et al. TETyper: a bioinformatic pipeline for classifying variation and genetic contexts of transposable elements from short-read wholegenome sequencing data. Microb Genom. 2018;4 Available from: https:// doi.org/10.1099/mgen.0.000232

32. Arthur M, Molinas C, Depardieu F. Characterization of Tn1546, a Tn3-related transposon conferring glycopeptide resistance by synthesis of depsipeptide peptidoglycan precursors in Enterococcus faecium BM4147. J Bacteriol Am Soc Microbiol. 1993;175:117-27.

33. Ondov BD, Treangen TJ, Melsted $\mathrm{P}$, Mallonee AB, Bergman NH, Koren S, et al. Mash: fast genome and metagenome distance estimation using MinHash. Genome Biol. 2016;17:132

34. Galata V, Fehlmann T, Backes C, Keller A. PLSDB: a resource of complete bacterial plasmids. Nucleic Acids Res. 2019;47:D195-202.

35. Csardi G, Nepusz T, Others. The igraph software package for complex network research. InterJournal, Complex Systems 2006;1695:1-9.

36. Pritchard L, Cock P, Esen Ö. pyani v0. 2.8: average nucleotide identity (ANI) and related measures for whole genome comparisons. 2019; Available from: https://zenodo.org/record/2584238\#.X-HWo3VKjSQ

37. Clewell DB, Weaver KE, Dunny GM, Coque TM, Francia MV, Hayes F. Extrachromosomal and mobile elements in enterococci: transmission, maintenance, and epidemiology. In: Gilmore MS, Clewell DB, Ike Y, Shankar $\mathrm{N}$, editors. Enterococci: from commensals to leading causes of drug resistant infection. Boston: Massachusetts Eye and Ear Infirmary; 2014.

38. Sullivan MJ, Petty NK, Beatson SA. Easyfig: a genome comparison visualizer. Bioinformatics. 2011:27:1009-10.

39. Altschul SF, Gish W, Miller W, Myers EW, Lipman DJ. Basic local alignment search tool. J Mol Biol. 1990;215:403-10.

40. Guy L, Kultima JR, Andersson SGE. genoPlotR: comparative gene and genome visualization in R. Bioinformatics. 2010;26:2334-5.

41. Yu G, Smith DK, Zhu H, Guan Y, Lam TT-Y. ggtree: an r package for visualization and annotation of phylogenetic trees with their covariates and other associated data. McInerny G, editor. Methods Ecol Evol 2017;8:28-36.

42. Argimón S, Abudahab K, Goater RJE, Fedosejev A, Bhai J, Glasner C, et al. Microreact: visualizing and sharing data for genomic epidemiology and phylogeography. Microb Genom. 2016;2:e000093.

43. Arredondo-Alonso S. Gitlab repository with an RMarkdown document integrating all the code and plasmid sequences required to reproduce the results presented in this manuscript. Gitlab. 2020; Available from: https:// gitlab.com/sirarredondo/vancomycin_dissemination.

44. Freitas AR, Tedim AP, Francia MV, Jensen LB, Novais C, Peixe L, et al. Multilevel population genetic analysis of vanA and vanB Enterococcus faecium causing nosocomial outbreaks in 27 countries (1986-2012). J Antimicrob Chemother. 2016;71:3351-66.

45. Wardal E, Kuch A, Gawryszewska I, Żabicka D, Hryniewicz W, Sadowy E. Diversity of plasmids and Tn1546-type transposons among VanA Enterococcus faecium in Poland. Eur J Clin Microbiol Infect Dis. 2017;36:313-28.

46. Cheng L, Connor TR, Sirén J, Aanensen DM, Corander J. Hierarchical and spatially explicit clustering of DNA sequences with BAPS software. Mol Biol Evol. 2013;30:1224-8.

47. Fernández-García L, Blasco L, Lopez M, Bou G, García-Contreras R, Wood T, et al. Toxin-antitoxin systems in clinical pathogens. Toxins. 2016;8 Available from: https://doi.org/10.3390/toxins8070227.

48. Hegstad K, Mikalsen T, Coque TM, Werner G, Sundsfjord A. Mobile genetic elements and their contribution to the emergence of antimicrobial resistant Enterococcus faecalis and Enterococcus faecium. Clin Microbiol Infect. 2010; 16:541-54.

49. Di Sante L, Morroni G, Brenciani A, Vignaroli C, Antonelli A, D'Andrea MM, et al. pHTß-promoted mobilization of non-conjugative resistance plasmids from Enterococcus faecium to Enterococcus faecalis. J Antimicrob Chemother. 2017;72:2447-53
50. Brodrick HJ, Raven KE, Harrison EM, Blane B, Reuter S, Török ME, et al. Whole-genome sequencing reveals transmission of vancomycin-resistant Enterococcus faecium in a healthcare network. Genome Med. 2016;8:4.

51. Carter GP, Buultjens AH, Ballard SA, Baines SL, Tomita T, Strachan J, et al. Emergence of endemic MLST non-typeable vancomycin-resistant Enterococcus faecium. J Antimicrob Chemother. 2016:71:3367-71.

52. Howden BP, Holt KE, Lam MMC, Seemann T, Ballard S, Coombs GW, et al. Genomic insights to control the emergence of vancomycin-resistant enterococci. MBio. 2013;4 Available from: https://doi.org/10.1128/mBio. 00412-13.

53. Abdelbary MHH, Senn L, Greub G, Chaillou G, Moulin E, Blanc DS. Wholegenome sequencing revealed independent emergence of vancomycinresistant Enterococcus faecium causing sequential outbreaks over 3 years in a tertiary care hospital. Eur J Clin Microbiol Infect Dis. 2019;38:1163-70.

54. Lanza VF, Baquero F, de la Cruz F, Coque TM. AcCNET (Accessory Genome Constellation Network): comparative genomics software for accessory genome analysis using bipartite networks. Bioinformatics. 2017;33:283-5.

55. Corel E, Lopez P, Méheust R, Bapteste E. Network-thinking: graphs to analyze microbial complexity and evolution. Trends Microbiol. 2016;24:224-37.

56. Bernard G, Greenfield P, Ragan MA, Chan CX. k-mer similarity, networks of microbial genomes, and taxonomic rank. mSystems. 2018;3. Available from: https://doi.org/10.1128/mSystems.00257-18

57. Acman M, van Dorp L, Santini JM, Balloux F. Large-scale network analysis captures biological features of bacterial plasmids. Nat Commun. 2020;11:2452.

58. Harris PNA, M WA. Beyond the core genome: tracking plasmids in outbreaks of multidrug-resistant bacteria. Clinical Infectious Diseases. 2020. Available from: https://doi.org/10.1093/cid/ciaa052

59. Arredondo-Alonso S, Willems RJ, van Schaik W, Schürch AC. On the (im) possibility of reconstructing plasmids from whole-genome short-read sequencing data. Microbial Genomics Microbiology Society. 2017;3 Available from: http://www.microbiologyresearch.org/content/journal/mgen/10.1099/ mgen.0.000128.

60. Orlek A, Stoesser N, Anjum MF, Doumith M, Ellington MJ, Peto T, et al. Plasmid classification in an era of whole-genome sequencing: application in studies of antibiotic resistance epidemiology. Front Microbiol. Frontiers; 2017;8:182.

61. Neumann B, Bender JK, Maier BF, Wittig A, Fuchs S, Brockmann D, et al. Comprehensive integrated NGS-based surveillance and contact-network modeling unravels transmission dynamics of vancomycin-resistant enterococci in a high-risk population within a tertiary care hospital. PLoS One. 2020;15:e0235160.

62. Arredondo-Alonso S, Top J, McNally A, Puranen S, Pesonen M, Pensar J, et al. Short-read sequencing data of 309 VRE isolates and associated set of 26 complete vanA plasmid sequences. European Nucleotide Archive at EMBL-EBI under accession number PRJEB28495. European Nucleotide Archive. 2020. Available from: https://www.ebi.ac.uk/ena/browser/view/ PRJEB28495

\section{Publisher's Note}

Springer Nature remains neutral with regard to jurisdictional claims in published maps and institutional affiliations.

Ready to submit your research? Choose BMC and benefit from:

- fast, convenient online submission

- thorough peer review by experienced researchers in your field

- rapid publication on acceptance

- support for research data, including large and complex data types

- gold Open Access which fosters wider collaboration and increased citations

- maximum visibility for your research: over $100 \mathrm{M}$ website views per year

At $\mathrm{BMC}$, research is always in progress.

Learn more biomedcentral.com/submission 